\title{
IV. Versuche über die Wirkung der heissen Luft nach Weigert bei Larynxtuberculose.
}

\author{
Von Dr. A. Nykamp in Leiden.
}

Es sei mir gestattet, kurz iiber das Resultat der Experimente zu berichten, die ich mit einem meiner Schüler Herrn Huber N ood, über die Heissluftbehandlung nach Weigert's Methode angestellt habe. Herr Huber Nood hat alle Details der von uns behandelten Fälle in seiner Dissertation veröffentlicht, in welcher er Näheres über Körpertemperatur, Pulsfrequenz, Frequenz der Respiration, Körpergewicht, Untersuchung der Sputa u. s. w. mittheilt.

Wir wählten nur Fälle von Larynxtuberculose, da wir uns dachten, dass, wenn in der That die Temperatur der eingeathmeten Luft (im Falle dieselbe in die Lungenalveolen eingedrungen wäre) noch hoch genug wäre, sterilisirend oder vernichtend auf die Tuberkelbacillen an diesen Stellen einzuwirken, diese Wirkung unsomehr die Schleinhaut des Larynx treffen wïrde, wenn dieselbe in Folge von Tuberculose erkrankt wäre.

Indem wir genau nach Weigert's Vorschrift nusere Fälle behandelten, versuchten wir auf experimentellem Wege eine Erklärung, wie es möglich sei, dass eine Schleimhaut so hohe Temperatur ertragen könne, ohne versengt zu werden oder ohne zn verbrennen.

In erster Linie muss darum folgende Frage beantwortet werden: Wenn das Thermometer am Weigert'schen Apparat z. B. 250 bis $300^{0} \mathrm{C}$ zeigt, wie hoch ist dann die Temperatur der eingeathmeten Luft, nachdem sie durch das kupferne Ansatzrohr in der Mundhöhle angelangt und alsdann in die Luftwege aufgenommen worden ist? Und wie gross ist der Temperaturunterschied in der Mundhöhle, dem Larynx und in den Bronchien? Zur Beantwortung dieser Fragen unternahmen wir Folgendes:

I. Statt des gewöhnlichen Mundstïckes nahmen wir eiue Glasröhre, woran sich am Ende ein gerade aufstehendes Röhrchen befand, in welchem ein Thermometer angebracht werden konnte.

Ausserdem brachten wir über dem Ausathmungsventil ein zweites Thermometer an, so dass dieses die Temperatur der ausgeathmeten Luft angeben konnte. War nun der Apparat genügend erhitzt (was wir statt mit der grossen Spiritusflamme von Weigert, mit einer Flehscher'schen Gasflamme thaten), und inspirirte einer von uns die trockene Luft, die nach dem Thermometer über dem Apparate eine Temperatur von $260^{\circ} \mathrm{C}$ haben sollte, dann stand das Thermometer, das sich am Ende der Glasröhre gerade vor dem Mundstïcke und $17 \mathrm{~cm}$ vom Inspirationsventil entfernt befand, $\mathrm{nur}$ a uf $80^{\circ} \mathrm{C}$, während das Thermometer über dem Exspirationsventil einen Stand von $60^{\circ} \mathrm{C}$ erreichte, was also die Temperatur der ausgeathmeten Luft vorstellen muss.

II. Wurde der Apparat auf die gewöhnliche Weise erhitzt, und zeigte das Thermometer über dem Apparat eine Temperatur von $150^{\circ} \mathrm{C}$ an, dann erreichte das Thermometer über dem Exspirationsventil einen Stand von $50^{\circ} \mathrm{C}$, ohne dass irgend welche Luft durch den Apparat strömte, da daran nicht eingeathmet wurde.

Hieraus ergiebt sich also: 1) dass bei der Inspiration von Luft, die bis zu $260^{\circ} \mathrm{C}$ erhitzt zu sein scheint, in einer Entfernung von ungefähr $17 \mathrm{~cm}$ in einer gläsernen Röhre eine Abkühlung bis zu $80^{\circ} \mathrm{C}$ stattfindet, und 2) dass die eigene Wärme der kupfernen Röhren ein wichtiger Factor ist für die Erreichung des hohen Thermometerstandes, ohne dass noch das Durchströmen der Luft. und die Erhitzung derselben die Ursache der Steigerung zu sein braucht.

III. Als wir eine an Larynxtuberculose leidende Kranke mit sehr frequentirter Respiration anf die gewöhnliche Weise einathmen liessen, betrug die Temperatur der eingeathmeten Luft nach dem Thermometer am Apparate $220^{\circ} \mathrm{C}$, während das über dem Ventil befindliche Thermometer $50^{\circ} \mathrm{C}$ als Temperatur der ausgeathmeten Luft anzeigte.

Ein männliches Individuum mit Larynxtuberculose, das 4-5 Respirationen per Minute zählte. inhalirte Luft aus dem Apparate, für die das Thermometer $265^{\circ} \mathrm{C}$ anzeigte, während die Temperatur der ausgeathmeten Luft nach der soeben beschriebenen Messung $60^{\circ} \mathrm{C}$ betrug.

Wenn es wirklich wahr wäre, dass die eingeathmete Luft eine Temperatur von 50 oder $60^{\circ}$ gehabt hätte, dann würde man mit Recht erwarten können, dass, wenn die Schleimhaut anf die Dauer gegen derartige Temperaturen Stand hielte, diese Methode imstande sein wïrde, vernichtend oder sterilisirend auf die Tuberkelbacillen einzuwirken, da diese bekanntlich einer niedrigeren Temperatur bedürfen, um existiren oder sich fortpflanzen zu können. Vergleichen 
wir jedoch dieses Resnltat mit demjenigen, welches wir beim $z$ weiten Experimente erzielten, wobei das Thermometer über dem Exspirationsventil eine fast ebenso hohe Temperatnr anzeigte, ohne dass am Apparate eingeathmet wurde, dann kann der erreichte Thermometerstand nie die genane Temperatnr der ansgeathmeten Lnft angeben, wenn am Apparate eingeathmet wird.

IV. Um nun zn nutersnchen, wie hoch die Temperatnr in der Mundhöhle sei, führten wir einen gebogenen Thermometer durch den nnteren Nasengang nud das Cavnm pharyngo-nasale in den Pharynx ein. Die Nasengänge wurden daranf sorgfältig abgeschlossen, damit keine Vermischnng mit kalter Lnft stattfinden sollte, nud nnn wnrde Luft, deren Temperatur $265^{\circ} \mathrm{C}$ betragen sollte, eingeathmet. Das Thermometer im Pharynx zeigte aber nur eine Temperatnr von $55^{\circ} \mathrm{C}$, ein Unterschied also von $210^{\circ} \mathrm{C}$.

V. Es blieb also nur noch übrig, die Temperatnr der Lnft in dem Larynx und in den Bronchien zn bestimmen.

Bei den zn diesem Zweck angestellten Experimenten narkotisirten wir jnnge Hnnde mit Morphinm nnd Chloral. Um den Kopf des Thieres wurde eine genan passende, nndnrchdringbare nud mit einem Mnndstïck versehene Maske befestigt, so dass dasselbe nngezwnngen durch die Nase athmen kounte.

Wir hatten zuerst die Nasengänge verstopft nnd danach das Mnndstück des Apparates im Manle befestigt nud mit Gnmmibandagen nmwickelt, nm einer Vermischnng mit kalter Lnft vorznbengen. Alsbald aber zeigten sich dann Ersticknngssymptome, weil die Znnge nach hinten znrücksinkt; bei der Befestignng der Znnge nach anssen jedoch ist die Respiration sehr gehemmt, da ein Hund fast ansschliesslich dnrch die Nase athmet.

Dann wnrde eine sehr niedrige Tracheotomie gemacht, wobei gesorgt wnrde, dass die Oeffnnng in der Trachea gerade genügend war, ein sehr dünnes gebogenes Thermometer nach der Bifnrcation der Bronchien einznführen. Die Mnskel-, sowie die Hantwnnde wnrden sorgfältig genäht. nnd das Thier konnte anf diese Weise fortathmen.

Die Temperatur in der Trachea anf der Höhe der Bifnrcation betrng $37^{\circ} \mathrm{C}$. Daranf wurde das Mnndstück in der Maske an den Apparat befestigt, nnd das Thier athmete Lnft ein, deren Temperatur $160^{\circ} \mathrm{C}$ betragen sollte. Ein Thermometer wnrde über dem Exspirationsventil angebracht. Nachdem das Thier reichlich eine halbe Stnnde eingeathmet, und das Thermometer einen Stand von $200^{\circ}$. C anfweist, also das Thier eine Luft, die bis $200^{\circ} \mathrm{C}$ erhitzt sein soll, einathmet, zeigt das Thermometer in der Trachea einen Stand von $36,3^{0} \mathrm{C}$, nnd das Thermometer n̈ber dem Exspirationsventil $55^{\circ} \mathrm{C}$. Die Temperatur der ansgeathmeten Lnft würde also $20^{\circ} \mathrm{C}$ höher sein, als die Temperatnr der Lnft in der Trachea ïber der Bifurcation der Bronchien.

VI. Wenn das Experiment anf dieselbe Weise vorgenommen, aber eine sehr hohe Tracheotomie gemacht nnd ein dünneres gebogenes Thermometer dnrch die Wunde nach oben geschoben wurde, so dass die Quecksilbersänle gerade nuter die Stimmbänder zn liegen kam, und die Temperatur der eingeathmeten Lnft $190^{\circ} \mathrm{C}$ betragen sollte, dann war die Temperatnr im Larynx gerade nnter den Stimmbändern $36^{\circ} \mathrm{C}$, die der ansgeathmeten Lnft $48^{\circ} \mathrm{C}$, nnd die Temperatnr des Thieres (im Anns gemessen) $34,2^{\circ} \mathrm{C}$. (Diese niedrige Körpertemperatur wurde durch die sehr tiefe Narkose vernrsacht.)

Ans diesen Experimente könnte man also schliessen, dass bei der Einathmnng von Lnft, die bis zn $1900 \mathrm{C}$ erhitzt sein soll, in dem Larynx eines. Hnndes die Temperatnr ein paar Grade höher ist, als in einem anderen Hohlranm des Körpers.

VII. Wenn ein normales oder krankes Individnum am Apparate heisse Lnft, z. B. von $250-280^{\circ} \mathrm{C}$ einathmet, nnd man bringt über dem Exspirationsventil eine lange gläserne Röhre an, dann sieht man alsbald, wie schon nach wenigen Respirationen die Röhre beschlagen ist, nnd wie die Fenchtigkeit sich bald daranf in grossen Tropfen, ja sogar in einem Wasserstrählchen ansscheidet. Im Anfang klagt der Einathmende über eine gewisse Trockenheit im Munde, die jedoch bald verschwindet. Hierans erhellt, dass, wenn anch die eingeathmete Lnft absolnt trocken ist, die ansgeathmete Lnft sehr mit Wasserdampf geschwängert ist.

VIII. Wenn in die bereits beschriebene, an der knpfernen Leitnngsröhre befestigte gläserue Röhre sehr fenchte Stückchen Schwamm eingeführt werden, nnd in dem gerade anfstehenden Röhrchen ein Thermometer befestigt wird, während die Ein- nnd Ansathmnng mittels eines kleinen Blasebalges stattfindet, dann zeigt Jetzteres Thermometer nngefähr $200^{\circ} \mathrm{C}$ weniger als dasjenige, welches die Temperatnr der trockenen Lnft angeben mnss. Eine imitirte Einathmung von nur wenigen Minnten genügt, die fenchten Stückchen Schwamm zn trocknen nud $\mathrm{zn}$ versengen.

Die Schlüsse, welche wir also mit Recht ans den beschriebenen Experimenten ziehen können, sind folgende:

1. Die Luft, welche mittels des Weigert'schen Apparates eingeathmet wird, wenn derselbe nach seiner Vorschrift erhitzt worden ist, scheint viel höher erhitzt $\mathrm{zn}$ sein, als es in Wirklichkeit der Fall ist, da die Temperatur der kupfernen Leitnngsröhre, in welcher der Thermometer sich befindet, $\mathrm{nm}$, wie es heisst, die Temperatnr der eingeathmeten Lnft zn bestimmen, den Stand des Thermometers erhöht.

2. Wenn anch die Temperatur der eingeathmeten Lnft sehr hoch ist, ja sogar mehrere Grade höher, als die Temperatnr, bei welcher Tuberkelbacillen bestehen oder sich fortpflanzen können, dann wird doch noch die Lnft nnterwegs, d. h. in der Mnndhöhle nnd in dem Pharynx, schon nm so viel Grade abgekühlt, dass dieselbe in dem Laryux nnd in den Bronchien nur etwas höher ist oder nur gleichkommt der in einem anderen Ranme des Körpers ge. messenen Temperatnr.

3. Die Ursache dieser Abkühlnng liegt in der Verdampfnng der grossen Quantität Wasser, die während der Einathmnng der heissen trockenen Lnft in der Mnndhöhle und im Pharynx der Schleimhant entzogen wird, eine Qnantität Wasser, welche dnrch die Circulation in den Lymph- nnd Blntgefässen immer anf's neue ersetzt wird.

Was das Resultat betrifft, das wir durch die Anwendung der Weigert'schen Methode bei Fällen von Larynxtuberculose erzielten, so kann ich mittheilen, dass ein günstiger Einfluss weder anf den localen Process, noch anf den allgemeinen Znstand der Patienten wahrgenommen worden ist. Nnr das wurde erreicht, dass wäh. rend der Einathmnng eine gewisse Lnngengymnastik ansgeübt wird. Die Kranken sind gezwnngen, einige Stunden täglich regelmässig Ein- und Ansathmnugsbewegnngen zn machen, wordurch eine bessere nnd reichliche Ventilation der Iuftwege stattfindet. Doch eine derartige Lnftoymnastik kann man anch anf einfachere nnd billigere Weise veranstalten. In dem Znstande also, in dem wir die Lnngentnberculose gewöhnlich beobachten, ist die Anwendnng der Weigert. schen Methode nnserer Ansicht nach vou durchans keinem Nntzen, weil das Gewebe. in welchem die Tnberkelbaillen, nnd zwar in der Tiefe desselben, anwesend sind, nie bis zn dem Grade erhitzt werden kann, ohne das Gewebe selbst nnd damit anch die pathogenen Bacterien, znm Nachtheile des ganzen Organismns, zn tödten.

Wir möchten daher vor der Anwendnng einer Methode warnen, von der anf experimentellem Wege sich zeigt, dass sie anf falscher Grundlage bernht, nnd bei deren Anwendnng bis jetzt, soweit mir wenigstens bekannt ist, keine sichere Heilnng oder Bessernng an bacillärer Phthisis constatirt worden ist. 